\title{
Intelligent Visualization System Analysis of Tunnel Monitoring
}

\author{
Li-bin ${ }^{1, a}$ \\ ${ }^{1}$ School of Civil and Enviromental Engineering, University of Science and Technology Beijing, Haidian, \\ Beijing 100083, China \\ a1055568768@qq.com
}

Keywords: tunnel monitoring, visualization, intelligent correlation, application

\begin{abstract}
In order to ensure the reliability and safety of the tunnel monitoring, this paper designs and realizes the general intelligent monitoring system for city tunnels, designs the overall structure of the visualization monitoring system, the processing algorithm of the database development module and the man-machine visualization interactive method, studies the historical data accumulated by the system, and gets the relations among the tunnel data. The application result shows that this system meets the demand for the tunnel information construction, and is characterized by quickness, convenience and practicality.
\end{abstract}

\section{Introduction}

With the rapid development of the transportation construction in China, the tunnel engineering construction is rapidly developing, and all kinds of accidents and geological disasters happen frequently. The real-time monitoring on the tunnel construction site can effectively prevent all kinds of accidents and geological disasters. The traditional monitoring method only focuses on the data storage or the two-dimensional monitoring. The data display is inflexible. The image display is not intuitive. The probability of query is poor. All of these greatly affect the response and decision-making efficiency of the administrative staff. The tunnel plays an important role in the transportation. The tunnel construction presents a rapid development trend. The properties of the underground tunnel show that some problems need to be solved in the construction process, including structure monitoring, gas monitoring, geological monitoring, and engineering process plan management. It is a complex process. The tunnel monitoring requires high standards and a long time. The measuring items are many and the data statistics is complex. Under many circumstances, the monitoring environment in tunnels is tough, which makes the traditional monitoring methods not meet ideal requirements. Especially after the tunnel completion, it is difficult to obtain the complete information of the tunnels via the manual monitoring. China has a short history in the tunnel monitoring researches, the technological level lags behind developed countries, and the popularity of intelligence is not high. Many factors result in the current situation, including the limited input cost, backward technologies, and slow development of the tunnel monitoring development. Therefore, a new intelligence monitoring method is helpful for the settlement of difficulties in the tunnel monitoring.

According to the database, VTK high-performance visualization development technology and the integration difference analysis module, this paper studies and sets up the tunnel monitoring intelligent visualization system. This system can manage the monitoring information effectively, exactly and intuitively.

\section{Overall Architecture Design of the Tunnel Visualization System}

The tunnel monitoring system is developed for ensuring the tunnel safety and stable operation. The main purpose is to monitor the internal environment quality in the tunnel, control the internal equipment, including the draught fan, lighting, license lamps and water pimp and other controllable equipment, and provide some services, such as fire alarm, video surveillance, broadcasting, voice communication and so on. The whole system includes hardware devices, signal transmission, 
network structures, workstations, servers, monitoring and control system software. The upper monitoring system is operated under the platform of Windows and uses the interchanger to build the local area network. All workstations are distributed in the same LAN and adopt the optical fiber to transmit the signal. The lower monitoring equipment use PLC to do monitoring. The main system structure of the whole system is shown as Figure 1:

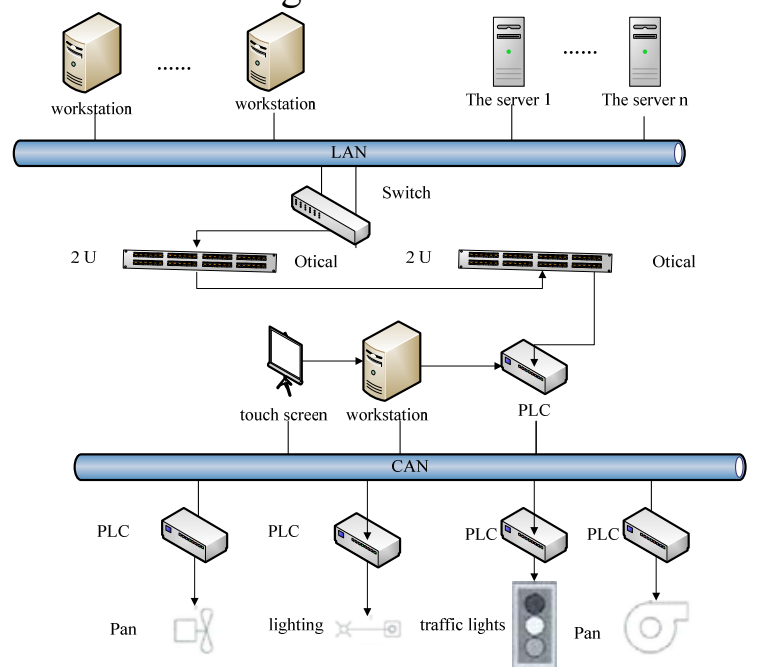

Figure 1 System Architecture

The monitoring system consists of many subsystems, including power monitoring system, draught fan monitoring system, lighting monitoring system, license lamp monitoring system, fire alarm system, video monitoring system, and water pump monitoring system. The listed monitoring subsystems here include all the tunnel monitoring systems, which consist of the tunnel monitoring system. The universal intelligence monitoring system discussed in this paper doesn't cover video, water pump, voice and telephone systems. The related subsystem integration will be discussed in Section Two of Chapter Four in detail. Figure 2 is the subsystem structure graph of the tunnel monitoring system: power monitoring subsystem, fan monitoring subsystem, lighting monitoring subsystem, license lamp monitoring subsystem, fire alarm subsystem and environmental monitoring subsystem are integrated into an integrated monitoring system. Different systems will work in different workstations.

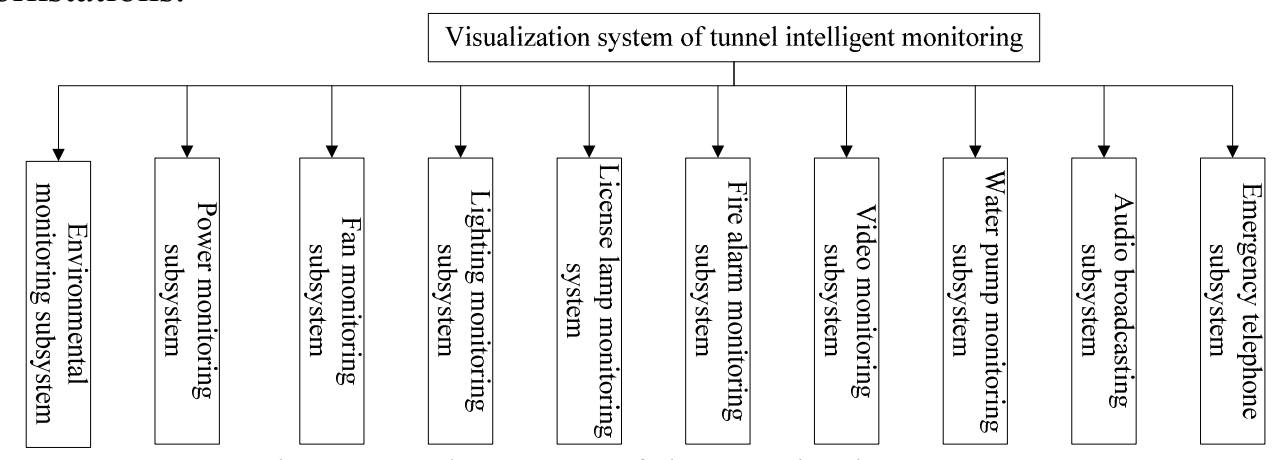

Figure 2 Subsystems of the Monitoring System

\section{Analysis of Key Technologies in the System Development}

\section{Database Development Module Design}

This system uses the VS2005 as the development environment, C\# as the development language and the dynamic link libraries of VTK.net. The database operation module uses the winforms provided by the .net framework database, which provides the visual U1 interface for the end-user. In this module, the user can add, delete, modify and check the database through the operation interface. The operation process of the database operation module is shown in Figure 3: 


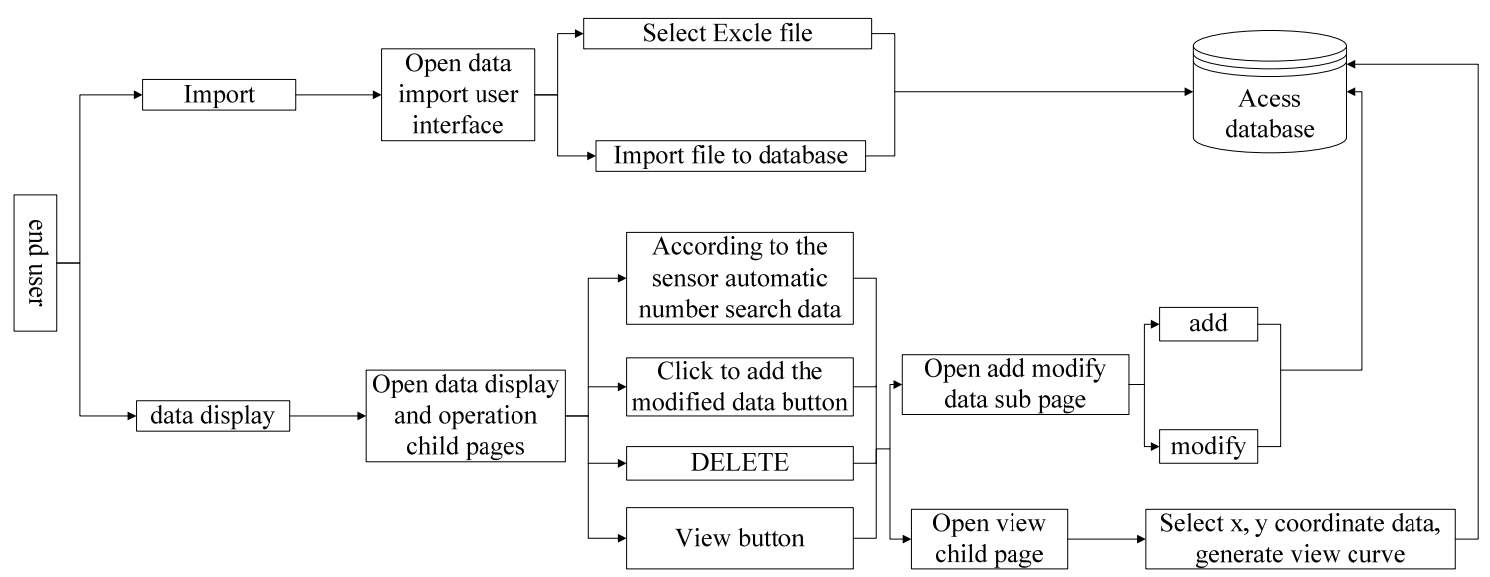

Figure 3 Operation Process of the Database Operation Module

The database operation module is the core module of maintaining and processing the data provided by this system for the user. This module is the presentation layer if the uppermost layer among the three-tier architecture. The presentation layer refers to the UI. Its main function is to provide the interaction between the application program and the user, and help the user meet his demand through the operation buttons on the UI. Because the system is three-tier architecture, the user just needs to click the code in the event index part and achieves the interaction with the database through the intermediate affair layer.

This system will call the Dundas chart controls and produce the data curve of $\mathrm{x}, \mathrm{y}$ axis for the user. The user can clearly understand the corresponding relation of the data, and it is convenient to analyze the previous interaction.

\section{Man-machine Interaction Visual Design}

Because the manual drawing will result in some errors for the coordinate displace of data points and stress value, the final contour line and cloud chart are not so accurate. The visualization module of the system adopts the graph visualization fragment database provided by VTK.net so that the original data measured in the tunnel engineering is converted into the graph and image on the computer screen.

The process of the visualization procedure is: take the data network as the Souree source, convert it into the data object model, pass the vtkContourFilier, connect the even points of the data network, produce the contour line, take the contour line as the input of the vtkPolyDataM, pass the channel flow of vtkPolyDataM, and the original input data will be converted into the geometric graphic elements for drawing. In the visualization module, in order to mark the corresponding relations in color between the scalar value and the contour line, the vtkscalarBarAetor object is realized. The vtkscalarBarAetor object provides the contrast between RGB color table and the scalar value through vtk okUpTable and shows the colored scalar range. After preparing the graph and image displayed in the VTK visualization window, it is necessary to establish the pipeline in the VTK visualization display scene.

\section{Intelligence Correlation Analysis of the Tunnel Data}

The data pretreatment can greatly improve the quality of the data and reduce the time of data mining, because the pretreatment can help the initial stage of data mining delete some gibberish, which will consume much time at a later stage. In addition, the deletion of dirty data will make the result of data mining more reliable and play decision-making and prediction roles. Figure 4 shows the whole data pretreatment process: 


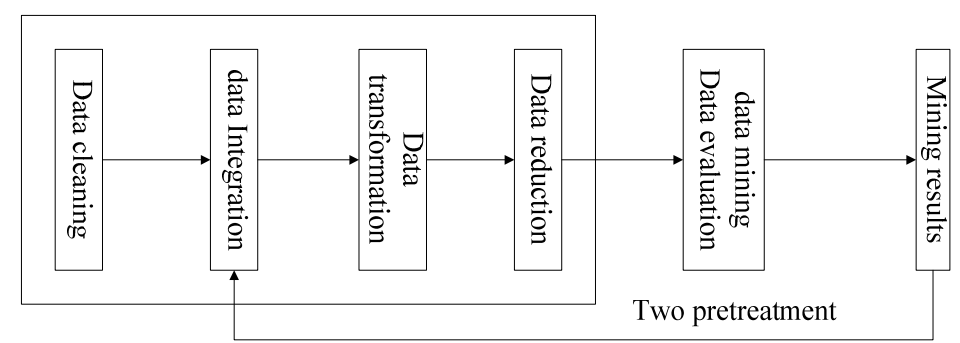

Figure 4 Data Pretreatment Process

This paper adopts the mathematical statistics method and conducts the data statistical analysis. According to the data distribution result, choose the reasonable range of all variables. The reasonable minimum and maximum values of all variable obtained from the database are shown in the following table: all the data beyond this range are taken as dirty data, which will be processed directly in this paper.

Table 1 Reasonable Value of Variables

\begin{tabular}{llll}
\hline Variable & Maximum & Average Value & Minimum \\
\hline ZCO & 1.56 & 0.89 & 0.72 \\
ZVI & 6.1 & 1.61 & 0.92 \\
ZFS & 6.37 & 2.74 & 0.07 \\
ZNGQ & 130.1 & 8.75 & -20.01 \\
ZSD & 72.87 & 48.02 & 10.97 \\
ZWD & 34.2 & 11.1 & 5.38 \\
ZCJ_SD & 126 & 77.5 & 0 \\
ZCJ_CLL & 70 & 18 & 0 \\
\hline
\end{tabular}

The data prediction and processing in the sample is based on Formula (1), and the data will be deleted and dealt with after getting correlation coefficient.

$$
r_{X y}=\frac{\sum_{i=1}^{n}\left(X_{i}-\bar{X}\right)\left(Y_{i}-\bar{Y}\right)}{\sqrt{\sum_{i=1}^{n}\left(X_{i}-\bar{X}\right)^{2}} \sqrt{\sum_{i=1}^{n}\left(Y_{i}-\bar{Y}\right)^{2}}}
$$

The range of $r$ is $[-1,1]$. When it is the positive correlation, $r>0$; otherwise, $r<0$. The value of $|r|$ presents a directly proportional relationship. In this paper, choose $r=0.6$ as the standard to get rid of some data.

The Apriori algorithm makes use of two properties of frequent item sets. That is to say, the superset of any non-frequent item set cannot be a frequent item set; the subset of any frequent item set must be a frequent item set. Suppose that $n$ items are non-frequent item sets, and then the superset of $n+1$ item in this set is also a non-frequent item. Thus, the Apriori algorithm is produced. The steps of the Apriori algorithm are: first, scan all the affair items of the affair data, get the frequent 1-item set L1 according to the minimum support, and get the candidate frequent 2-item set C2 through self-join L1. Next, scan all the affair items of the database once again, delete the items with the minimum support and produce L2. In a similar way, get all the frequent k-item set by the way of the iteration method until the self-join $\mathrm{Lk}$ produces an empty $\mathrm{Ck}+1$, and produce the frequent item sets, including $\mathrm{L} 1, \ldots \mathrm{Lk}$.

1 Scan the affair database to get the candidate set without covering the repeating elements and traversal affair data. The affair with the candidate items is added into the affair mark list of candidate items. And calculate the total amount, which will be taken as the support of this candidate item. Do cutting according to the minimum support and keep the item set with the satisfying minimum support. Finally, get the frequent 1 -item set L1.

$2 \mathrm{~L} 1$ carries out the self-join operation and produce the candidate frequent 2-item set $\mathrm{C} 2$. And then, the elements in the $\mathrm{C} 2$ candidate items produce their own affair lists through self-join (that is to calculate the public affair of the two candidate items), and do counting. Compare the affair list counting and the minimum support and add the candidate with the satisfying support into L2. At the same time, set up the L2 mapping address table A2 in the form of array. Store the address field of different header elements in L2 into A2. 
3 Produce Ck through Lk-1 self-join. If the subset of candidate items in Ck is not the subset of Lk-1 (this operation is quickly conducted through Ak-1 mapping address table), delete this item. Otherwise, link the affair list of candidate items and count the affair lists. Add the candidate item with the satisfying minimum support into Lk. Judge whether the header element of this candidate item exists in the mapping address table. If not, add it into Ak.

4 Repeat the third step, until $\mathrm{Ck}$ is empty.

5 Repeat the third step, until $\mathrm{Ck}$ is empty.

\section{System Visualization Verification}

This paper chooses the Baijiapo Tunnel, located among the Fangjiaquan Village, Yuzhong County, Lanzhou. The tunnel barrel passes through the higher terraces of Yellow River and loess ridge, which have a salient relief. And the relative height difference is up to over 300 . The valley on the surface is growing, and the cut $\mathrm{J}$ is relatively deep. Only the surface of the entrance and exit and the valleys have thick pellet soil. Other surfaces are covered by loess. The surface of the mountain slop has rare vegetation. The mileage of the tunnel is DK7+284-DK10+382, 3098m. It is a double-track tunnel, and the minimum burial depth is $35 \mathrm{~m}$, and the maximum is $300 \mathrm{~m}$. The algorithm is realized by means of VS2008 development tool and C++ language. The program will get the data directly from the data table Apriori, and the minimum support will be set according to the total data amount for mining. The frequent item sets and their support in the processing process will be stored through the hash table. All the frequent item sets will be finally obtained through iteration with $\mathrm{N}$ times and the processing process of connecting step and cutting step. The support of the correlation rule can be calculated in the following way:

$$
\operatorname{Support}(X \Rightarrow Y)=\frac{\operatorname{Sup}(X \cup Y)}{\| D} \quad(2)
$$

Confidence can be calculated in the following equation through the frequent item set:

$$
\text { Comfidence }(X \Rightarrow Y)=\frac{\operatorname{Sup}(X Y Y)}{\operatorname{Sup}(X)}
$$

Sup (CO_LAVI_MAFS_LAGQ_MACLL_LACS_H) $=9461$;

$\operatorname{Sup}(\mathrm{VI}-\mathrm{MAFS}$ LAGQ_MACLL_L八CS_H $)=9567$;

$|\mathrm{D}|=88388$;

Calculation:

Support $=9461 / 88388=10.7 \% \quad$ confidence $=9461 / 9567=98.9 \%$

In the above way, the correlation rule of $\mathrm{CO}-\mathrm{L}$ is as follows:

VI_MAFS_LAGQ_MACLL_LACS_H $=>$ CO_L [support $=10.7 \%$, confidence $=98.9 \%$ ]

Open the function interface of the monitoring measurement and import the file of the monitoring measurement in the construction process of Fusong Tunnel. After choosing the import file, the import speed can be seen below the data display column. Figure 5 is the relation curve between deformation and time.

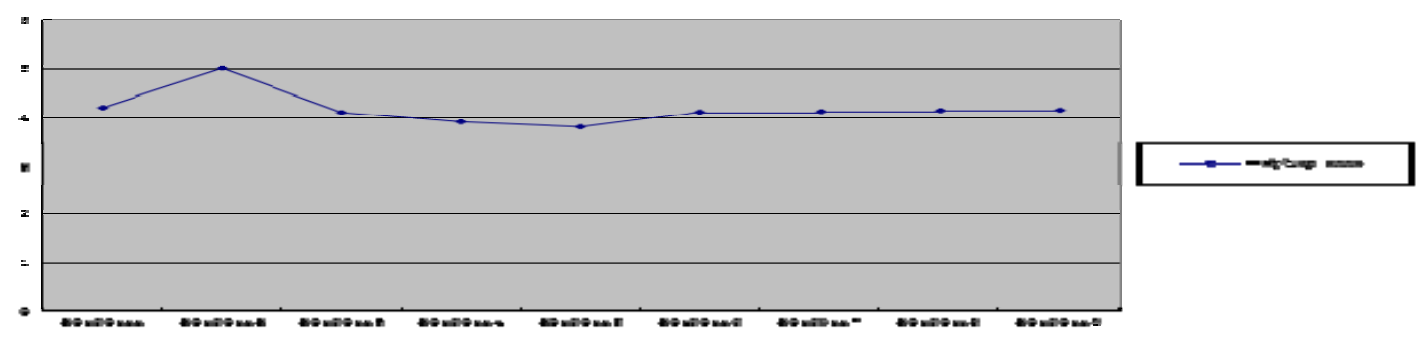

Figure 5 Deformation Curve

As shown in Figure 6, the analog results are shown through the VTK visualization procedure. According to the displacement and stress contour map, the largest horizontal displacement occurs around the tunnel with a symmetrical distribution and $2.272 \mathrm{~cm}$. the real monitoring displacement is $2.201 \mathrm{~cm}$. The relative error is $3.25 \%$. The analog results can, to some extent, analyze and predict 
the deformation resulting from the tunnel excavation.

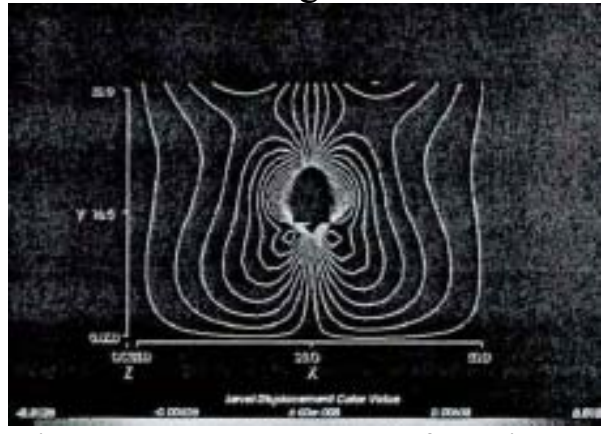

Figure 6 Contour Map of Horizontal Displacement

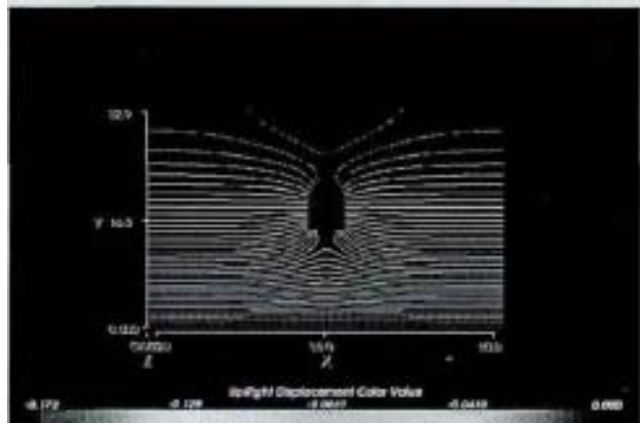

Figure 7 Contour Map of Vertical

Displacement

\section{Conclusion}

With the rapid development of the urban transportation, the urban tunnels are becoming more and more. If the tunnel monitoring system doesn't have the universality for different tunnels, a large number of human resources will be wasted. For the other development direction of the tunnel monitoring system, the software engineering is the core. The preliminary integration develops the intelligent visualization system in tunnel monitoring, which has been applied into the engineering. Researches have been done in the tunnel incidence relations, the association rule algorithm is adopted for data analysis, and the valuable research results have been achieved. The application results show that this system meets the demand for the tunnel information construction, and is characterized by quickness, convenience and practicality.

\section{References}

[1] Yu Wentao, Zhang Shaoli. Design and Application of the Highway Tunnel Monitoring System. Journal of Henan Radio \& TV University, 2005, PP55-57.

[2] Skinner Eugene and G.W.Lanyon. Measurement of in-situ stress in weak rock at Mont Terri Rock Laboratory. Switzerland. International Journal of Rock Mechanics \& Mining Sciences 40(2003), PP 1077-1088..

[3] Wang Jun, Xia Caichu, Zhu Hehua. Field Monitoring and Analysis of Asymmetric Multi-arch Tunnels. Chinese Journal of Rock Mechanics and Engineering. Vol 23(2):2004, PP267-271

[4] Zhong Denghua, Liu Donghai. Theoretical Method and Application of the Engineering Visual Computer-aided Design. Beijing: China Water\&Power Press, 2004.

[5] Zhou Guohao. Long-distance real-time monitoring System Development of the Laser Tunnel Surrounding Rock Displacement. Xi'an University of Science and Technology, 2004, p.6-9.

[6] Xiao Long, Zhang Cui, Lu Kaining. The Improvement Design Based on the Trend and Rising Cloud Security Method. China Education Network, Vol 02, 2011, p.86-87.

[7] Dai Gaofei, Ying Song, Xia Caichu, Mao Haihe. The New Construction Monitoring of the Expressway Tunnel. Journal of Chongqing University,2004,27(2)21 